\title{
Application of cell-free DNA for genomic tumor profiling: a feasibility study
}

\author{
Lise B. Ahlborn ${ }^{1,2}$, Kristoffer S. Rohrberg ${ }^{1}$, Migle Gabrielaite ${ }^{2}$, Ida V. Tuxen ${ }^{1}$, \\ Christina W. Yde ${ }^{2}$, Iben Spanggaard ${ }^{1}$, Eric Santoni-Rugiu ${ }^{3}$, Finn C. Nielsen ${ }^{2}$, Ulrik \\ Lassen $^{1}$, Morten Mau-Sorensen ${ }^{1, *}$, Olga Østrup ${ }^{2, *}$ \\ ${ }^{1}$ The Phase I Unit, Department of Oncology, Rigshospitalet, Copenhagen University, Copenhagen, Denmark \\ ${ }^{2}$ Center for Genomic Medicine, Rigshospitalet, Copenhagen University, Copenhagen, Denmark \\ ${ }^{3}$ Department of Pathology, Rigshospitalet, Copenhagen University, Copenhagen, Denmark \\ *These authors contributed equally to this work \\ Correspondence to: Lise B. Ahlborn, email: lise.barlebo.ahlborn@regionh.dk \\ Keywords: cfDNA; liquid biopsy; genomic profile; biopsy; WES \\ Received: October 24, $2014 \quad$ Accepted: January 17, $2019 \quad$ Published: February 15, 2019 \\ Copyright: Ahlborn et al. This is an open-access article distributed under the terms of the Creative Commons Attribution License \\ 3.0 (CC BY 3.0), which permits unrestricted use, distribution, and reproduction in any medium, provided the original author and \\ source are credited.
}

\section{ABSTRACT}

Purpose: Access to genomic tumor material is required to select patients for targeted therapies. However, tissue biopsies are not always feasible and therefore circulating cell-free DNA (cfDNA) has emerged as an alternative. Here we investigate the utility of cfDNA for genomic tumor profiling in the phase I setting.

Study design: Peripheral blood was collected from patients with advanced solid cancers eligible for phase I treatment. Patients failing the initial tissue biopsy due to inaccessible lesions or insufficient tumor cellularity $(<10 \%)$ were included in the study. Genomic profiling of cfDNA including whole exome sequencing (WES) and somatic copy number alterations (SCNAs) analysis (OncoScan).

Results: Plasma cfDNA was pro- and retrospectively profiled from 24 and 20 patients, respectively. The median turnaround time was 29 days $(N=24$, range 1387 days) compared to tissue re-analyses of median 60 days $(\boldsymbol{N}=6$, range 29-98). Selected cancer-associated alterations (SCAAs) were identified in $70 \%(31 / 44)$ of patients, predominantly by WES due to the low sensitivity of OncoScan on cfDNA. Primarily, inaccessible cases of prostate and lung cancers could benefit from cfDNA profiling. In contrast, breast cancer patients showed a low level of tumor-specific cfDNA which might be due to cancer type and/or active treatment at the time of plasma collection.

Conclusion: Plasma cfDNA profiling using WES is feasible within a clinically relevant timeframe and represents an alternative to invasive tissue biopsies to identify possible treatment targets. Especially, difficult-to-biopsy cancers can benefit from cfDNA profiling, but tumor tissue remains the gold standard for molecular analyses.

\section{INTRODUCTION}

Knowledge of the genomic makeup of tumors is essential for cancer diagnosis, prognosis, and selection of treatment. With the great advancements in the field of precision medicine, genotype-directed therapy is becoming a standard tool for stratification of oncological patients. For instance, HER2 gene expression and EGFR mutation status are used to guide treatment for breast and non-small cell lung cancers (NSCLC), respectively $[1,2]$. Ideally, fresh tissue is used to characterize the tumor but often archival material such as formalin-fixed paraffin-embedded (FFPE) tissue is used introducing several problems. First, archival tissue might not represent 
the current malignancy due to clonal evolution of the disease over time and in response to previous therapies $[3,4]$. Second, DNA from FFPE tissue are often highly fragmented influencing downstream analyses $[5,6]$. Although tissue biopsies, either fresh or archival, represent standard for molecular testing, poor quality or inadequate quantity of tissue and DNA is often challenging besides the discomfort and risks of complications related to biopsy procedures. In NSCLC, tissue biopsies are unusable in $20-30 \%$ of patients [7], highlighting the need for an alternative source of tumor material.

Circulating cell-free DNA (cfDNA) has been widely investigated as a potential surrogate for tissue biopsies for non-invasive assessment of tumor-related genomic alterations as circulating tumor DNA (ctDNA) can be identified in cfDNA. Recently, FDA approved EGFR-mutation testing based on cfDNA for treatment stratification of NSCLC [8]. Due to the rapid development of NGS technology, it is now possible to characterize the molecular profile of cfDNA [9, 10] but no previous studies have investigated comprehensive cfDNA profiling in a prospective setting. The focus of whole exome sequencing (WES) studies has been on characterizing tumor heterogeneity and resistance $[11,12]$ and only few prospective studies have included cfDNA analyses, focusing mainly on treatment monitoring using small genepanels [13]. In a prospective study, Kaisaki et al., showed that targeted-sequencing of cfDNA in early stage lung cancers, was a valuable alternative to tissue in a diagnostic setting due to high concordance of tumor mutations between tissue and cfDNA [14]. Little has been reported on turnaround time of cfDNA analyses in a prospective setting, but results from retrospective cohorts have reported analysis times between 10-15 days [10, 15, 16]. Furthermore, studies characterizing somatic copy number alterations (SCNAs) in cfDNA have been sparse [17].

We performed a feasibility study including a proand retrospective cohort of patients eligible for phase I treatment where tissue was inaccessible for biopsy or the biopsy was too low in tumor cell content. Genomic cfDNA profiling included WES and SCNAs analyses (OncoScan). The aim of the study, was to investigate whether tumorspecific DNA alterations could be identified in plasma cfDNA and whether the analysis could be performed within a time frame relevant in a clinical setting.

\section{RESULTS}

\section{Patient characteristics}

A total of 118 advanced cancer patients were prospectively enrolled in the CoPPO project from January to August 2018 (Figure 1). Of these, 24 patients (17\%) underwent cfDNA analysis either because the tumor tissue was inaccessible for biopsy $(N=9$, Cohort 1$)$ or the obtained tissue biopsy had low tumor cellularity ( $N=15$, Cohort 2$)$.
Various cancer types were included, the most represented being colorectal $(N=5)$ and prostate cancer $(N=4)$ (Table 1). Six patients had subsequently a successful re-biopsy performed and three cases had archival FFPE tissue analyzed. Twenty patients were included in a retrospective cohort (Cohort 3), selected from the total CoPPO cohort as illustrated in Supplementary Figure 1. Eight patients from Cohort 3 were re-biopsied and genomic reports on tumor tissue were obtained. Across cohorts, an equal distribution of males (50\%) and females (50\%) were included and $41 \%$ $(18 / 44)$ of the patients received treatment at the time of plasma collection (Supplementary Table 1). Furthermore, most patients had multiple metastatic sites with the most common biopsy site being liver, representing 67\% (22/33) of the biopsy sites (Supplementary Table 1). Radiological assessment of the overall tumor burden was not available at the time of plasma sampling. Assessment of overall tumor burden is not a standard procedure in the CoPPO study and would not impact the enrollment of patients.

\section{Feasibility of genomic cfDNA profiling}

\section{Method efficacy}

Cell-free DNA was successfully extracted from all prospectively enrolled patients with concentrations ranging from 1.5 to $120 \mathrm{ng} / \mathrm{ml}$ plasma (median $8.0 \mathrm{ng}$ / ml) (Supplementary Table 2). The retrospective cohort was selected based on available cfDNA (Supplementary Figure 1) with concentrations from 2.2 to $181.5 \mathrm{ng} /$ $\mathrm{ml}$ plasma (median $26.2 \mathrm{ng} / \mathrm{ml}$ ) (Supplementary Table $2)$. Whole exome sequencing was performed on all patients $(N=44)$ with a median overall average coverage of $186 \mathrm{x}$ (interquartile range $\mathrm{IQR}=273$; $\mathrm{Q} 1=90.80$; $\mathrm{Q} 3=364.30$ ) and median 10x coverage on 94\% of the exome (interquartile range $\mathrm{IQR}=0.0135$; $\mathrm{Q} 1=0.9344$; $\mathrm{Q} 3=0.9479$ ) (Supplementary Figure 2 and Supplementary Table 2), similar to previous studies $[18,19]$.

OncoScan array used varying cfDNA input concentrations ( $\geq 5 \mathrm{ng}$ ) depending on the amount remaining after WES analysis (Supplementary Table 2). The median input was $20 \mathrm{ng}$ cfDNA (range 7-80 ng). The OncoScan analysis was achieved on 40 out of 44 patients, as four samples had insufficient input material for full genomic profiling and therefore WES was prioritized. Of the 40 samples, 8 samples failed the analysis due to quality issues of either cfDNA $(N=1, \mathrm{P} 42)$ or OncoScan array $(N=$ 7, "Failed (suboptimal quality)" in Supplementary Table 4). Noticeably, cfDNA concentration or input amount did not seem to affect ctDNA detection by OncoScan or WES although the small sample size could influence this result ( $P>0.05$, t-test, unequal variance).

\section{Turnaround time}

The median turnaround time for cfDNA profiling in Cohort 1 and 2 was 29 days (range 13-87 days), defined as the median time from failed tissue biopsy 
Table 1: Patient characteristics

\begin{tabular}{|c|c|c|c|}
\hline Characteristic & $\begin{array}{l}\text { Prospective Cohorts } 1+2 \\
(N=24)\end{array}$ & $\begin{array}{c}\text { Retrospective Cohort } 3 \\
(N=\mathbf{2 0})\end{array}$ & $\begin{array}{l}\text { All patients } \\
\qquad(N=44)\end{array}$ \\
\hline Age (median, range) & $62(36-82)$ & $64(26-75)$ & $64(26-82)$ \\
\hline \multicolumn{4}{|l|}{ Gender } \\
\hline Male & 12 & 10 & $22(50 \%)$ \\
\hline Female & 12 & 10 & $22(50 \%)$ \\
\hline \multicolumn{4}{|l|}{ Tumor origin } \\
\hline Colorectal & 5 & 10 & 15 \\
\hline Breast & 3 & 5 & 8 \\
\hline Prostate & 4 & 1 & 5 \\
\hline Endometrial & 2 & 0 & 2 \\
\hline Head and neck & 1 & 1 & 2 \\
\hline Bile duct & 1 & 2 & 3 \\
\hline Lung (NSCLC) & 3 & 0 & 3 \\
\hline Ovarian & 1 & 0 & 1 \\
\hline Other $^{\mathrm{A}}$ & 4 & 1 & 5 \\
\hline \multicolumn{4}{|l|}{ Cohorts } \\
\hline 1 (Prospective, no biopsy) & 9 & - & - \\
\hline 2 (Prospective, failed biopsy) & 15 & - & - \\
\hline 3 (Retrospective) & - & 20 & - \\
\hline $\begin{array}{l}\text { Number of patients with } \geq \\
1 \mathrm{SCAA}^{\mathrm{B}}\end{array}$ & $21(88 \%)$ & $10(50 \%)$ & $31(70 \%)$ \\
\hline \multicolumn{4}{|l|}{ Turnaround time (days) } \\
\hline $\begin{array}{l}\text { cfDNA, } N=24 \text { (median, } \\
\text { range) }\end{array}$ & $29(13-87)$ & - & - \\
\hline Tissue, $N=6$ (median, range) & $60(29-98)$ & - & - \\
\hline \multicolumn{4}{|l|}{ Profiling on other materials } \\
\hline Tissue re-biopsy & 6 & 8 & 14 \\
\hline Archival FFPE tissue & 3 & 0 & 3 \\
\hline
\end{tabular}

${ }^{\mathrm{A}}$ Included: Mesothelioma $N=1$, Testicular $N=1$, Pancreatic $N=1$, Gastric $N=1$, Cervical $N=1$

${ }^{B} \mathrm{SCAA}$ : Selected cancer-associated alterations

until the genomic report was completed (Supplementary Figure 3). The analysis time decreased and stabilized during the study due to improvement and establishment of the cfDNA workup. A fresh tissue re-biopsy was obtained and successfully profiled for six patients in the prospective cohorts. The median turnaround time was 60 days (range 29-98) defined as the time from failed biopsy until completion of the genomic report on the re-biopsy (Supplementary Figure 3). Of note, inclusion of patients in Cohort 1 , where no biopsy could be obtained, commenced after the initial tests of the cfDNA pipeline.

\section{Detection of genomic tumor alterations in cfDNA}

A complete genomic cfDNA report including both WES and OncoScan (+/- SCAA) was achieved in $32 / 44$ cases (Figure 2). Whole exome sequencing was successfully performed in all 44 cases and at least one SCAA was identified in $68 \%$ (30/44 patients) of patients. The most frequently altered genes were $\operatorname{TP} 53(N=14)$, $A P C(N=9), \operatorname{KRAS}(N=4), \operatorname{ATR}(N=14)$ and PIK3CA $(N=3)$ (Figure 2A and Supplementary Table 3). A range of 30 different SCAAs was observed only once $(N=1)$. In $32 \%$ 
(14/44 patients), WES did not detect a SCAA with almost half of these being breast cancers including a large fraction of patients in active treatment at the time of blood collection (Figure 3). Significantly more breast cancers were observed in the -SCAA group compared to the +SCAA group from either WES or OncoScan ( $P=0.0048$, Firsher's exact test $)$, whereas the fractions in treatment were not significantly different between the two SCAA-groups $(P=0.098$, Firsher's exact test). OncoScan array was successfully performed on 32/44 samples with acceptable quality values, with $43 \%(N=19)$ showing a silent chromosomal profile and $30 \%(N=13 / 44)$ with detectable SCAAs (Figure 2B and Supplementary Table 4). The most common SCAA were deletion of either TP53 $(N=4), C D K N 2 A(N=3)$, or $A P C(N=2)$ and amplification of $A R(N=3)$ and $M Y C(N=2)$. Furthermore, bi-allelic loss of $C D K N 2 A$ was observed in two patients $(N=2)$. As previously mentioned, $18 \%(N=8)$ of the samples failed the analyses and $9 \%(N=4)$ were not analyzed due to limited cfDNA material (Figure 2B).
A SCAA was identified from either WES or OncoScan in $70 \%$ of the patients (31/44) involving all cancer types included in the study (Figure 3). This was lower than the overall CoPPO cohort (100\%, Figure 1), however expectable from the type of input material. Whole exome sequencing lead to detection of SCAAs in $95 \%$ of the tissue biopsies compared to $88 \%$ and $45 \%$ in cfDNA from the pro-and retrospective cohorts, respectively (Figure 1). Adding the OncoScan SCNA analysis to the cfDNA profiling did not increase the number of positive findings in the prospective cohorts and only included one more patient (P35, prostate cancer with $A R$ amplification) in the retrospective cohort.

\section{Comparing genomic profiles identified by WES in plasma and tissue DNA}

Fourteen re-biopsies and three archival FFPE samples were included in the study to compare the tumor

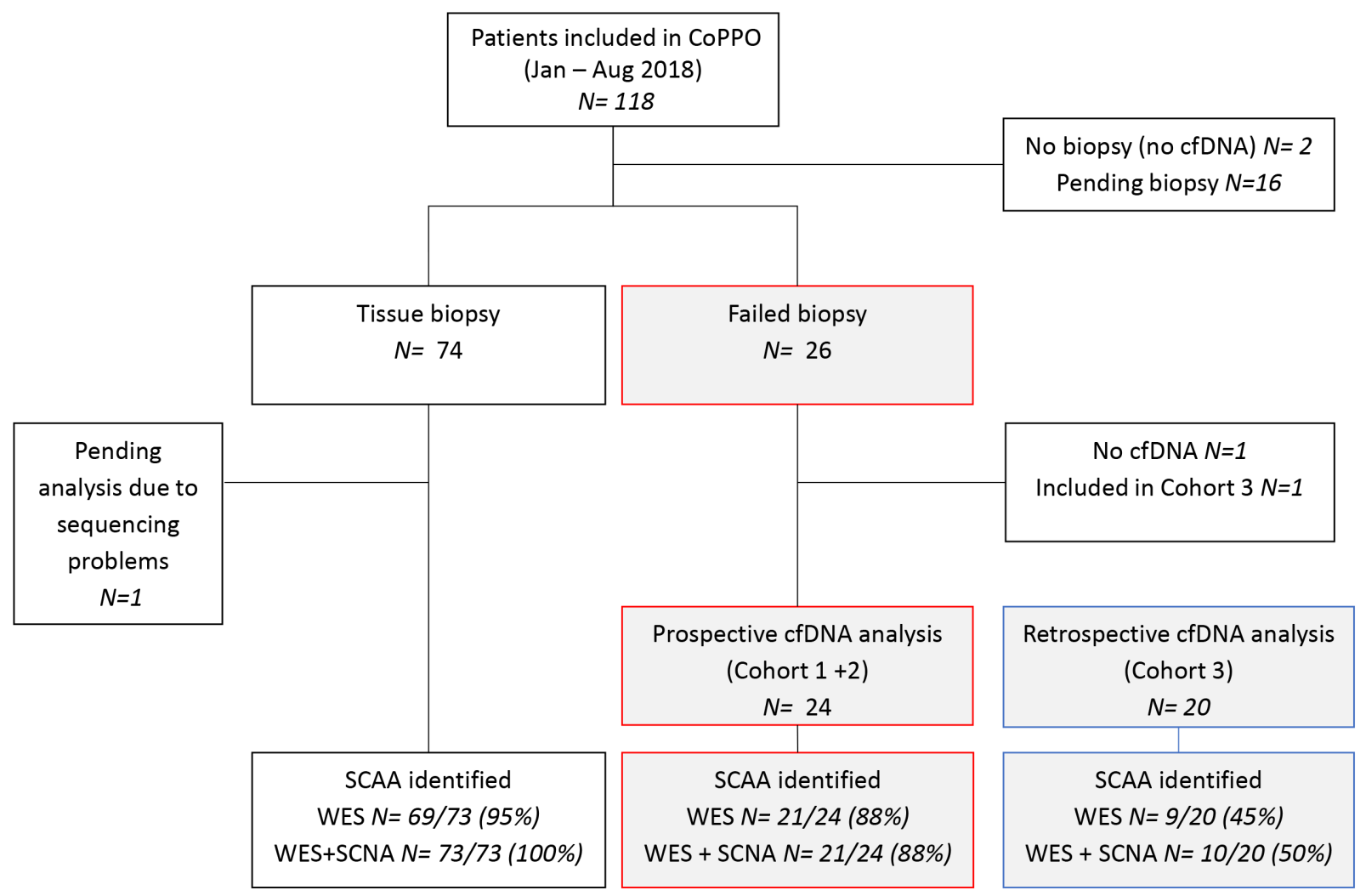

Figure 1: Genomic profiling of the CoPPO cohort within the study period. A total number of 118 patients were included in the CoPPO project from January to $1^{\text {st }}$ of August 2018. Eighteen patients were excluded because no plasma was collected for cfDNA analysis or tumor biopsies were pending. Tissue biopsies were usable for genomic profiling in 74 cases and a SCCA (selected cancer-associated aberrations) were identified in $95 \%$ and $100 \%$ of patients when using WES (whole exome sequencing) or WES+SCNA (somatic copy number alteration) analysis, respectively. The gray/red boxes indicate 26 patients with failed tissue biopsies either due to low tumor cell content $(<10 \%$, Cohort 2$)$ or to inaccessible tumors (Cohort 1). Two patients were excluded; one due to lack of cfDNA and the other was included in Cohort 3 due prolonged start of the cfDNA-pipeline due to implementation of the setup in the laboratory. Twenty-four patients were prospectively profiled based on plasma cfDNA and a SCAA was identified using WES alone or plus SCNA analysis in $88 \%$ of the patients. In the retrospective cohort (gray/blue boxes) a SCAA was found in $45 \%$ using WES and in 50\% when OncoScan analysis was included. $N$ indicate the number of patients in each group. 
tissue to cfDNA (total $N=17$ ). Nine of the sample-pairs were obtained in the prospective cohort. Two samplepairs could not be compared due to lack of detectable SCAAs (patient P38) and suboptimal quality of analysis (patient P18) and were excluded from the comparison (Supplementary Table 5). Shared SCAAs were observed in $73 \%(11 / 15)$ of patients from WES data. Private mutations were detected in 33\% (5/15) and 67\% (10/15) of plasma and tissue DNA samples, respectively (Table 2 and Supplementary Table 5).

\section{DISCUSSION}

In this study, we show that genomic tumor profiling of cfDNA was feasible in all 44 patients compared to only $25 \%$ of patients who were eligible for re-biopsy. Plasma cfDNA profiling thus represents a minimallyinvasive alternative when tissue biopsies are not available. Especially, within the group of patients who cannot be biopsied, the benefit of applying cfDNA into cancer diagnostics is undoubtable. We successfully detected SCAAs in cfDNA in $8 / 9$ patients in Cohort 1 primarily being NSCLC and prostate cancers with inaccessible thoracic and bone metastases, respectively. In all prospective prostate cancer samples, a SCAA was identified mainly being amplification of the $A R$ gene which is a well-known resistance mechanism in $50 \%$ of castration-resistant prostate cancers $[20,21]$. In these cancer types, the use of cfDNA for tumor profiling is of great importance as a growing number of targeted therapies and clinical trials are available for different molecular subtypes of prostate and lung cancers [22-24]. None of the 24 prospectively analyzed patients had tumor alterations identified in cfDNA that were actionable by an open clinical trial or off-label program at our institution at the time of analysis. This was in line with the general CoPPO cohort in which only $20 \%$ of patients $(101 / 500$ biopsied patients) received treatment based on tumor tissue profiling [25].
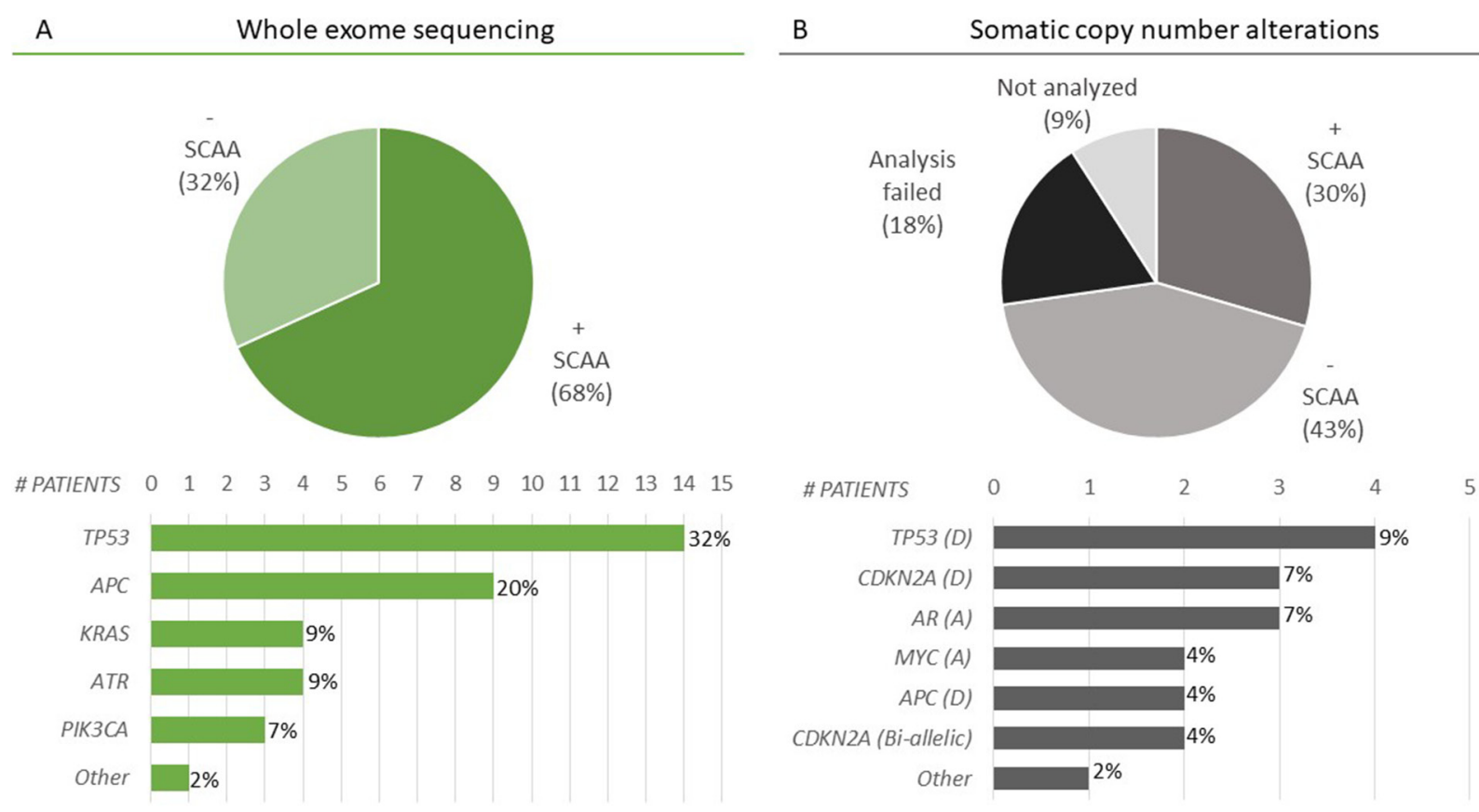

Figure 2: Selected cancer-associated alterations (SCAAs) identified in cfDNA. (A) WES was successfully performed on all cfDNA samples $(N=44)$. SCAAs were identified in $68 \%(30 / 44)$ of patients (+ SCAA). The most frequently altered genes were $T P 53$, $A P C, K R A S, A T R$, and PIK3CA. Furthermore, 30 different genes (NRAS, PIK3CG, BRAF, ATM, ARIDIA, AKT2, FGF10, PCDHB12, ACIN1, RAD21, RAD51C, MYO6, SMARCA5, RAB14, FANCD2, CHD7, PTEN, AR, MAP3K9, WT1, POLR3B, MAP2K2, HDAC9, SOX9, $M A P 2 K, S M A D 4, R A D 50, S M A R C C 2$, CHEK1, IDH1) where mutated in only a single patient each, indicated by Other. Information on the individual alterations are included in Supplementary Table 3. (B) Analysis of somatic copy number alterations (SCNA) by OncoScan identified SCAAs in $30 \%$ of patients (13/44) most often involving deletion $(D)$ or amplification $(A)$ of $T P 53(N=4)$, CDKN2A $(N=3), A R$ $(N=3), M Y C(N=2)$, or $A P C(N=2)$. Other included genes that were mutated in only a single patient being: CCND1 (A), KRAS $(A), J A K 2$ (A), MET (A), PTEN (bi-allelic). The bar plot, include only genes where a SCNA was identified together with a mutation in the same gene leading to both alleles affected. All SCNAs are reported in Supplementary Table 4. A silent chromosomal profile (- SCAA) was found in $43 \%(19 / 44)$ of patients. The analysis failed in $18 \%(8 / 44)$ of the cases due to suboptimal quality of the cfDNA $(N=1)$ or arrays $(N=7)$. In 4 cases $(9 \%)$, the amount of cfDNA was insufficient for analysis. 
Despite a small sample size, this study indicates, that some cancer types might be more suitable for cfDNA profiling than others. Surprisingly, only $2 / 8$ breast cancers had a SCAA identified by WES with mutation frequencies around 5\%. None of the samples showed a positive finding on OncoScan, despite the high prevalence of SCNAs in breast cancers, often involving deletion of $P I K 3 C A$ or amplification of $E R B B 2[26,27]$. These results could reflect a low fraction of plasma ctDNA in breast cancer patients that might be explained by the effect of active treatment at the time of plasma collection possibly increasing the level of normal cfDNA [28]. This was shown in a recent study of 210 patients with NSCLC, where ctDNA was detected in $43 \%$ of patients receiving systemic therapy at the time of plasma sampling and in $75 \%$ of patient not in therapy [29]. Moreover, the low ctDNA detection might also be related to a small tumor burden known to correlate with both cfDNA and ctDNA levels [30, 31]. We have not correlated our findings with tumor burden due to limited access to radiological data at the time of plasma collection. Furthermore, we did not include baseline RECIST measurements as these underestimated the actual tumor burden due to exclusion of non-target lesions [32].

Analysis of cfDNA immediately following failed biopsy, markedly decreased the time to completed genomic reports (median 29 vs 60 days), a key factor for timely allocation of treatment in the Phase1 setting.

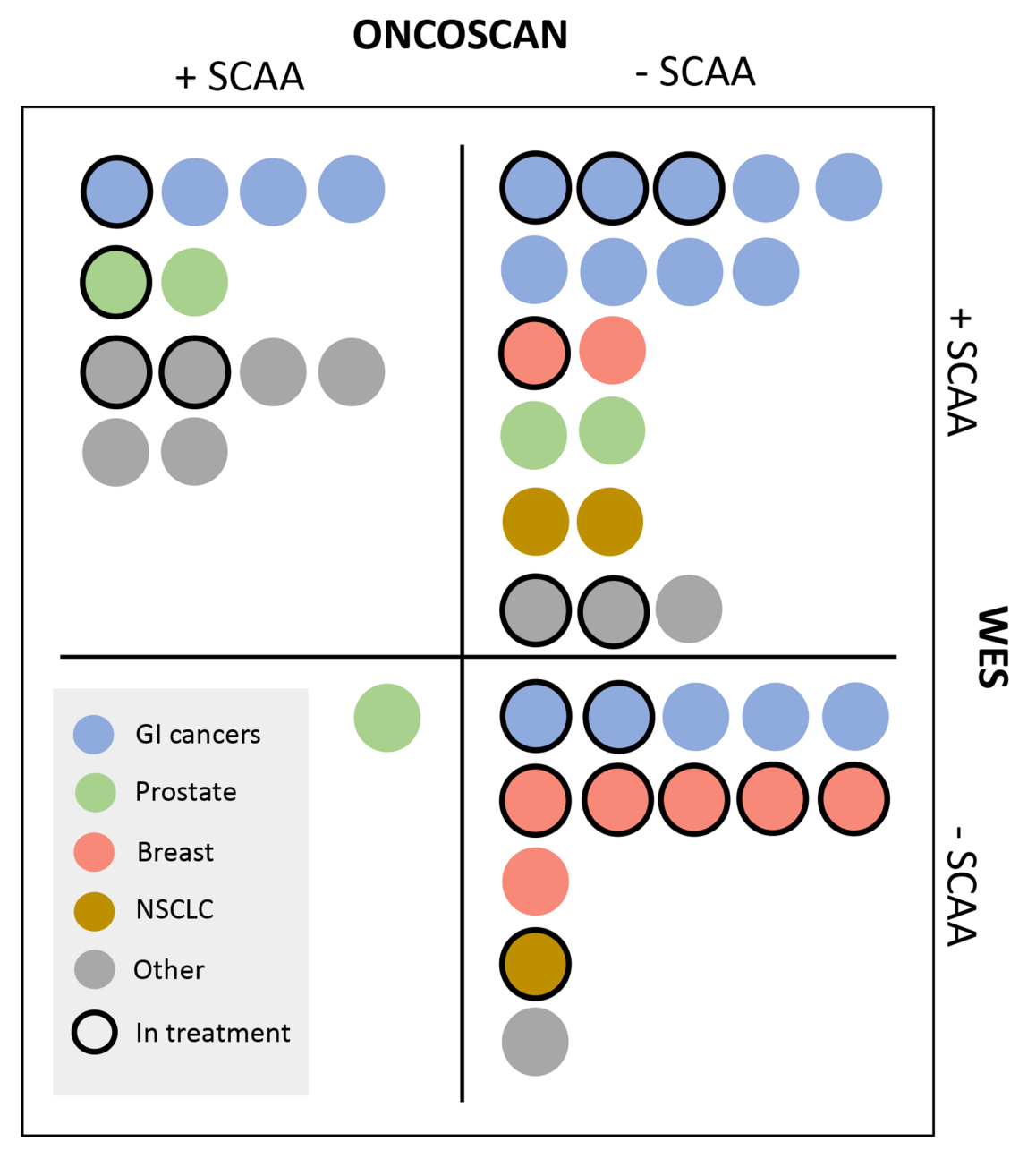

Figure 3: OncoScan and WES results indicated by cancer type and active treatment $(\boldsymbol{N}=44)$. Identification of a selected cancer-associated alteration (SCAA) from either OncoScan or WES is indicated by $+S C A A$. Negative reports from WES (no SCAA, Supplementary Table 3) and Silent profiles, Failed, plus No analysis from OncoScan analyses (Supplementary Table 4) are indicated as $-S C A A$. Cancer types represented $\geq 3$ times across cohorts, are color coded as shown in the legend. Gastrointestinal (GI) cancers included bile duct $(N=3)$ and colorectal cancers $(N=15)$. Cancer types "Other" included: Endometrial $N=2$, Gastric $N=1$, Ovarian $N=1$, Head and neck $N=2$, Mesothelioma $N=1$, Testicular $N=1$, Pancreatic $N=1$, Cervical $N=1$. Patients in active treatment at the time of plasma cfDNA collection are marked with a full-line border. Additional individual information is provided in Supplementary Table 1, 3, and 4. 
Table 2: Selected cancer-associated alterations (SCAAs) identified by WES in plasma and tissue DNA

\begin{tabular}{|c|c|c|c|c|}
\hline Patient ID (cohort) & Cancer subtype & Shared SCAAs & SCAAs in cfDNA & SCAAs in tissue \\
\hline P1 (2) & Endometrial & ATM, ARID1A, AKT2 & ATM, ARID1A, AKT2 & $\begin{array}{c}A T M, A R I D 1 A, A K T 2, \\
R A D 51 C\end{array}$ \\
\hline P2 (2) & Ovarian & TP53 & TP53, FGF10 & TP53 \\
\hline P4 (2) & Head and neck & TP53, RAD21 & TP53, RAD21 & $\begin{array}{c}\text { TP53, RAD21, } \\
P I K 3 C A, C D K N 2 A, \\
R E T\end{array}$ \\
\hline P6 (2) & Pancreatic & KRAS, TP53 & KRAS, TP53, FANCD2 & $K R A S, T P 53, A T R \times 2$ \\
\hline P8 (2) & Testicular & SMARCA5, RAB14 & $\begin{array}{c}S M A R C A 5, R A B 14 \\
\text { MYO6 }\end{array}$ & $\begin{array}{c}S M A R C A 5, R A B 14, \\
P O L R 3 A\end{array}$ \\
\hline P9 (1) & Prostate & TP53 & TP53 & TP53 \\
\hline $\mathrm{P} 13(1)^{\mathrm{F}}$ & Prostate & PTEN & PTEN, ATR & PTEN \\
\hline $\mathrm{P} 23(1)^{\mathrm{F}}$ & Lung (NSCLC) & $K R A S$ & KRAS, CHEK1 & $K R A S$ \\
\hline P37 (3) & Breast & $P I K 3 C A \times 2$ & PIK3CA $\times 2$ & $P I K 3 C A \times 2, T P 53$ \\
\hline P40 (3) & Colorectal & TP53 & TP53 & TP53, MET \\
\hline P41 (3) & Bile duct & $I D H 1, A T R$ & $I D H 1, A T R$ & $I D H 1, A T R$ \\
\hline
\end{tabular}

Alterations identified by whole exome sequencing (WES) in patients with SCAAs identified in plasma and tissue DNA $(N=11)$. Patients where mutations were only identified in either cfDNA or tissue DNA are shown in Supplementary Table 5. F FFPE material used for WES.

Even though, the time from failed biopsy until re-biopsy could be improved from the median 31 days observed here, cfDNA analysis would still be superior with respect to turnaround time. Furthermore, in some clinical settings, validation of potential treatment targets might be performed thus increasing the turnaround time concerning both tissue and cfDNA samples.

Despite all the advantages of cfDNA profiling, biopsy material remains the method of choice. The most obvious restriction of using cfDNA for genomic tumor profiling is the low tumor fraction $(0.01-10 \%)$ in cfDNA $[33,34]$, in combination with the limited sensitivity of the current methods. OncoScan analysis only identified SCAAs in $13 / 44$ patients most likely due to cfDNA input levels below the recommended $80 \mathrm{ng}$. In this small feasibility study, we could not assign any correlation between total cfDNA levels and the identification of ctDNA by either method $(P>0.05)$. However, increased cfDNA levels might reflect a high degree of normal tissue destruction potentially diluting the ctDNA signal leading to false negative ctDNA results. In addition, the highly fragmented nature of cfDNA could also affect method efficacy. Solutions adjusted to small fragment DNA sizes are being developed [35], together with SCNA assays optimized for cfDNA [17]. Furthermore, it has been shown that increasing the cfDNA input to $>20$ ng for WES analysis improved the detection of ctDNA [19].
Another challenge of using cfDNA for oncological diagnostics, is the intrapersonal tumor evolution and hereby, a high degree of tumor heterogeneity. In this study, $65 \%$ of the SCAAs identified by WES was shared between plasma and tissue and private mutations were detected in $33 \%$ and $67 \%$ of plasma and tissue, respectively. However, large comparative studies are needed to assess the concordance between tumor and plasma biopsies and ongoing studies of postmortem samples like the PEACE study (NCT03004755) might help clarifying this aspect. Alterations in cfDNA have been suggested to represent clonal alterations rather than subclonal [18], possibly explaining the lower number of SCAAs private to cfDNA compared to tissue observed here. The lack of complete concordance between cfDNA and tissue likely reflects: 1) That the clonal nature of the tumor is not captured by a single biopsy; 2) That some tumor clones shed little DNA, or the frequency of the alteration was below the cut-off of $5 \%$; Or 3 ) that the tumor evolves over time and in response to therapy, mainly important for the FFPE samples, representing archival material potentially years older than the cfDNA sample.

To our knowledge, this is the first study to test WES and SCNA analysis by OncoScan of cfDNA in a prospective trial. Previous studies have successfully used cfDNA for genomic profiling, but these studies have included only few patients [36] or not been clinically 
relevant due to the high level of sequencing coverage and costs [37]. Our feasibility study was limited in sample size and heterogeneity of cancer types. Furthermore, we tested only WES and OncoScan as these methods were available in our clinical setting. Indications related to the effect of cancer type and active therapy on cfDNA profiling needs further validation in larger and uniform cohorts.

In conclusion, genomic tumor profiling using plasma cfDNA constitutes an alternative when tissue biopsies are unavailable. Especially, WES identified SCAAs and potential treatment targets whereas SCNA analysis by OncoScan needs optimization for cfDNA. Advanced prostate and lung cancer patients can particularly benefit from cfDNA profiling contrary to breast cancers, where preselection based on treatment status should be considered. Finally, more SCAAs were identified in fresh tumor tissue highlighting that tissue is still the preferable material for genomic profiling due to the higher density of tumor DNA.

\section{MATERIALS AND METHODS}

\section{Patients}

Patients with metastatic solid tumors were recruited to the Phase I Unit at Rigshospitalet, Department of Oncology, Copenhagen University, as part of the Copenhagen Prospective Personalized Oncology study (CoPPO) project (NCT02290522)[25]. The study was conducted in accordance with the Declaration of Helsinki and written informed consent was obtained for all patients (Danish Ethical Committee, file number: 1300530). We included three cohorts: 1) A prospective cohort (Cohort 1; $N=9$ ) including patients with tumors inaccessible for biopsy due to location e.g. bone metastasis only, inaccessible thoracic lesions etc.; 2) A prospective cohort (Cohort 2; $N=15$ ) including patients whose initial tissue biopsy failed the genomic analyses due to low levels of tumor cells $(<10 \%)$; 3) A retrospective cohort (Cohort 3; $N=20$ ), consisting of patients whose initial biopsy failed and was unusable for genomic profiling. The inclusion period for Cohort 1 and 2 was from January to $1^{\text {st }}$ of August 2018.

\section{DNA collection and purification}

Two blood samples were collected from each patient, one for germline DNA (gDNA) analysis as described for the CoPPO study [25] and one for cfDNA analysis. Peripheral blood for cfDNA analysis was collected in cellstabilizing BCT-tubes (Streck Laboratories) as previously described [38] and extracted from $4 \mathrm{ml}$ plasma using the QIAsymphony Circulating DNA Kit (Qiagen) according to the manufacturer's instructions using an elution volume of $60 \mu \mathrm{l}$. Extracted cfDNA was quantified using a dsDNA
HS Assay Kit on a Qubit Fluorometer (Thermo Fisher Scientific) and subsequently stored at $-20^{\circ} \mathrm{C}$ until further use. For patients with a successful re-biopsy, fresh tumor tissue was collected in RNA-later as part of the CoPPO project. Archival FFPE tissue was included if available and DNA was extracted using the Gene Read DNA FFPE Kit (Qiagen).

\section{Genomic profiling}

DNA libraries were prepared from $10 \mathrm{ng}$ cfDNA or FFPE-DNA using the NEBNext Ultra II protocol (New England Biolabs) and hybridized using the MedExome capture panel (Roche) or Agilent SureSelect system, respectively. DNA libraries from gDNA or fresh tissue re-biopsies were generated from $500 \mathrm{ng}$ DNA using SureSelect (Agilent). All DNA libraries enriched for exonic sequences were then quantified and quality-controlled using a Qubit HS flourometer and the TapeStation 4200 High Sensitivity assay (Agilent). Finally, all libraries were sequenced as paired-end on the NextSeq or HiSeq sequencer (Illumina). Plasma cfDNA libraries were sequenced with an average coverage $>100 \mathrm{x}$ (Supplementary Figure 2).

Sequencing reads were mapped to the hg19/ GRCh37 reference genome using the CLC Workbench v.3.5.4. Tumor-specific variants were identified by excluding germline variants and requiring a variant to have 1 ) $\geq 10 x$ coverage; 2 ) forward/reverse balance $\geq$ 0.1 ; (3) tumor variant frequency $\geq 5 \%$. Variants were identified using the Ingenuity Variant Analysis Software (Qiagen) and selected cancer-associated alterations (SCAAs) were manually inspected and included in the final genomic reports comprising: 1) Previously reported cancer variants described in COSMIC [39], Ingenuity Knowledge database (Qiagen) or in published literature and 2) novel truncating alterations in tumor suppressor genes or missense alterations in known oncogenes predicted by in silico analysis [40] to be affecting protein function. The SCAA definition thus included class I to III variants according to the Tier classification [41]. The analysis excluded low quality and common variants $(>1 \%$ in the Exome Aggregation Consortium database (ExAC), 1000 Genomes Project or NHLBI Exome sequencing project (ESP)) and included non-synonymous alterations as well as splice site alterations $+/-2 b p$ from exon/intron boundaries.

Somatic copy number alterations (SCNA) were analysed using the OncoScan CNV Plus assay (ThermoFisher Scientific) with minimum input of $5 \mathrm{ng}$ cfDNA. The data was visually inspected and analysed using the Nexus Software v 8.0 (BioDiscovery). The analysis of SCNAs focused on detection of amplification (copy number $>5$ ), bi-allelic losses, deletions, and loss of heterozygosity (LOH). On fresh tissue re-biopsies, SCNAs were analyzed using CytoScanHD assay (Affymetrix) 
with DNA input of $250 \mathrm{ng}$ according to the manufactures instructions. Sensitivity of the applied methods were 5\% and $20-30 \%$ for WES and OncoScan, respectively. Hence a negative report does not necessarily indicate lack of tumor specific alterations or ctDNA.

\section{Statistical analyses}

A t-test was used to test, whether cfDNA concentration or DNA input affected the results from OncoScan analysis. Due to small sample sizes, Fishersexact test was performed to compare the number of breast cancer patients harboring a SCAA to those with no SCAA. A $P$-value $<0.05$ was considered statistically significant. Furthermore, descriptive statistics were used, including medians, ranges, and frequencies. All statistical analyses were done in $\mathrm{R}$ (version 0.99.903).

\section{Abbreviations}

cfDNA: cell-free DNA; ctDNA: Circulating tumor DNA; WES: whole exome sequencing; WGS: whole genome sequencing; SCNAs: somatic copy number alterations; FFPE: formalin-fixed paraffin-embedded; NSCLC: non-small cell lung cancers; NGS: next generation sequencing; CoPPO: Copenhagen Prospective Personalized Oncology study; gDNA: germline DNA; SCAA: selected cancer-associated alterations; ExAC: Exome Aggregation Consortium database; ESP: Exome sequencing project; IQR: Inter quartile range.

\section{Author contributions}

The study was designed and supervised by OØ, MMS and CWY. Analysis and interpretation of data was performed by LBA and $O \varnothing$ who also primarily wrote the manuscript. KSR, IS and MMS enrolled patients in the CoPPO study headed by FCN, ESR and UL. MG provided computational support related to the exome sequencing and IVT provided clinical data on the patients.

\section{ACKNOWLEDGMENTS}

We sincerely thank patients and staff at the Phase I unit and Centre for Genomic Medicine, Copenhagen University, Rigshospitalet. Especially, we thank the NGS and array technicians for indispensable help with tumor and plasma analysis.

\section{CONFLICTS OF INTEREST}

ES-R has received lecture honoraria from Pfizer, Novartis, Boehringer Ingelheim, and Takeda. MMS has received lecture honoraria and support to participate in scientific conference from Roche. KSR received support to participate in scientific conference from Roche and
Sanofi. All remaining authors have declared no conflicts of interest.

\section{FUNDING}

This study was supported by the Danish Cancer Society and the Danish Cancer Research Fund (Dansk Kræftforskningsfond). The sponsors had no role in study design, data analysis, or writing of the article.

\section{REFERENCES}

1. Slamon DJ, Leyland-Jones B, Shak S, Fuchs H, Paton V, Bajamonde A, Fleming T, Eiermann W, Wolter J, Pegram M, Baselga J, Norton L. Use of chemotherapy plus a monoclonal antibody against HER2 for metastatic breast cancer that overexpresses HER2. N Engl J Med. 2001; 344: 783-92. https://doi.org/10.1056/NEJM200103153441101.

2. Tsao MS, Sakurada A, Cutz JC, Zhu CQ, Kamel-Reid S, Squire J, Lorimer I, Zhang T, Liu N, Daneshmand M, Marrano P, da Cunha Santos G, Lagarde A, et al. Erlotinib in lung cancer - molecular and clinical predictors of outcome. N Engl J Med. 2005; 353: 133-44. https://doi. org/10.1056/NEJMoa050736.

3. Diaz LA Jr, Williams RT, Wu J, Kinde I, Hecht JR, Berlin J, Allen B, Bozic I, Reiter JG, Nowak MA, Kinzler KW, Oliner KS, Vogelstein B. The molecular evolution of acquired resistance to targeted EGFR blockade in colorectal cancers. Nature. 2012; 486: 537-40. https://doi.org/10.1038/ nature 11219.

4. McGranahan N, Swanton C. Biological and therapeutic impact of intratumor heterogeneity in cancer evolution. Cancer Cell. 2015; 27: 15-26. https://doi.org/10.1016/j. ccell.2014.12.001.

5. Gilbert MT, Haselkorn T, Bunce M, Sanchez JJ, Lucas SB, Jewell LD, Van Marck E, Worobey M. The isolation of nucleic acids from fixed, paraffin-embedded tissues-which methods are useful when? PLoS One. 2007; 2: e537. https:// doi.org/10.1371/journal.pone.0000537.

6. Kerick M, Isau M, Timmermann B, Sultmann H, Herwig R, Krobitsch S, Schaefer G, Verdorfer I, Bartsch G, Klocker H, Lehrach H, Schweiger MR. Targeted high throughput sequencing in clinical cancer settings: formaldehyde fixedparaffin embedded (FFPE) tumor tissues, input amount and tumor heterogeneity. BMC Med Genomics. 2011; 4: 68. https://doi.org/10.1186/1755-8794-4-68.

7. Vanderlaan PA, Yamaguchi N, Folch E, Boucher DH, Kent MS, Gangadharan SP, Majid A, Goldstein MA, Huberman MS, Kocher ON, Costa DB. Success and failure rates of tumor genotyping techniques in routine pathological samples with non-small-cell lung cancer. Lung Cancer. 2014; 84: 39-44. https://doi.org/10.1016/j. lungcan.2014.01.013.

8. U.S. Food and Drug Administration . Summary of safety and effectiveness data: cobas EGFR Mutation Test v2. 
September 28, 2016. http://www.accessdata.fda.gov/cdrh_ docs/pdf15/P150044B.pdf.

9. Schwaederle M, Husain H, Fanta PT, Piccioni DE, Kesari S, Schwab RB, Banks KC, Lanman RB, Talasaz A, Parker BA, Kurzrock R. Detection rate of actionable mutations in diverse cancers using a biopsy-free (blood) circulating tumor cell DNA assay. Oncotarget. 2016; 7:9707-17. https://doi.org/10.18632/oncotarget.7110.

10. Schwaederle M, Husain H, Fanta PT, Piccioni DE, Kesari S, Schwab RB, Patel SP, Harismendy O, Ikeda M, Parker BA, Kurzrock R. Use of Liquid Biopsies in Clinical Oncology: Pilot Experience in 168 Patients. Clin Cancer Res. 2016; 22: 5497-505. https://doi.org/10.1158/10780432.CCR-16-0318.

11. Chicard M, Colmet-Daage L, Clement N, Danzon A, Bohec M, Bernard V, Baulande S, Bellini A, Deveau P, Pierron G, Lapouble E, Janoueix-Lerosey I, Peuchmaur M, et al. Whole-Exome Sequencing of Cell-Free DNA Reveals Temporo-spatial Heterogeneity and Identifies TreatmentResistant Clones in Neuroblastoma. Clin Cancer Res. 2018; 24: 939-49. https://doi.org/10.1158/1078-0432. CCR-17-1586.

12. Toledo RA, Garralda E, Mitsi M, Pons T, Monsech J, Vega E, Otero A, Albarran MI, Banos N, Duran Y, Bonilla V, Sarno F, Camacho-Artacho M, et al. Exome Sequencing of Plasma DNA Portrays the Mutation Landscape of Colorectal Cancer and Discovers Mutated VEGFR2 Receptors as Modulators of Antiangiogenic Therapies. Clin Cancer Res. 2018; 24: 3550-9. https://doi.org/10.1158/1078-0432. CCR-18-0103.

13. Toledo RA, Cubillo A, Vega E, Garralda E, Alvarez R, de la Varga LU, Pascual JR, Sánchez G, Sarno F, Prieto SH, Perea S, Lopéz-Casas PP, López-Ríos F, Hidalgo M. Clinical validation of prospective liquid biopsy monitoring in patients with wild-type RAS metastatic colorectal cancer treated wi th FOLFIRI-cetuximab. Oncotarget. 2017; 8:35289-300. https://doi.org/10.18632/oncotarget.13311.

14. Kaisaki PJ, Cutts A, Popitsch N, Camps C, Pentony MM, Wilson G, Page S, Kaur K, Vavoulis D, Henderson S, Gupta A, Middleton MR, Karydis I, et al. Targeted NextGeneration Sequencing of Plasma DNA from Cancer Patients: Factors Influencing Consistency with Tumour DNA and Prospective Investigation of Its Utility for Diagnosis. PLoS One. 2016; 11: e0162809. https://doi. org/10.1371/journal.pone.0162809.

15. Schwaederle M, Chattopadhyay R, Kato S, Fanta PT, Banks KC, Choi IS, Piccioni DE, Ikeda S, Talasaz A, Lanman RB, Bazhenova L, Kurzrock R. Genomic Alterations in Circulating Tumor DNA from Diverse Cancer Patients Identified by Next-Generation Sequencing. Cancer Res. 2017; 77: 5419-27. https://doi.org/10.1158/0008-5472. CAN-17-0885.

16. Shu Y, Wu X, Tong X, Wang X, Chang Z, Mao Y, Chen X, Sun J, Wang Z, Hong Z, Zhu L, Zhu C, Chen J, et al. Circulating Tumor DNA Mutation Profiling by Targeted
Next Generation Sequencing Provides Guidance for Personalized Treatments in Multiple Cancer Types. Sci Rep. 2017; 7: 583. https://doi.org/10.1038/s41598-017-00520-1.

17. Van Roy N, Van Der Linden M, Menten B, Dheedene A, Vandeputte C, Van Dorpe J, Laureys G, Renard M, Sante T, Lammens T, De Wilde B, Speleman F, De Preter K. Shallow Whole Genome Sequencing on Circulating Cell-Free DNA Allows Reliable Noninvasive Copy-Number Profiling in Neuroblastoma Patients. Clin Cancer Res. 2017; 23: 630514. https://doi.org/10.1158/1078-0432.CCR-17-0675.

18. Adalsteinsson VA, Ha G, Freeman SS, Choudhury AD, Stover DG, Parsons HA, Gydush G, Reed SC, Rotem D, Rhoades J, Loginov D, Livitz D, Rosebrock D, et al. Scalable whole-exome sequencing of cell-free DNA reveals high concordance with metastatic tumors. Nat Commun. 2017; 8: 1324. https://doi.org/10.1038/s41467-017-00965-y.

19. Koeppel F, Blanchard S, Jovelet C, Genin B, Marcaillou C, Martin E, Rouleau E, Solary E, Soria JC, Andre F, Lacroix L. Whole exome sequencing for determination of tumor mutation load in liquid biopsy from advanced cancer patients. PLoS One. 2017; 12: e0188174. https://doi. org/10.1371/journal.pone.0188174.

20. Chen CD, Welsbie DS, Tran C, Baek SH, Chen R, Vessella R, Rosenfeld MG, Sawyers CL. Molecular determinants of resistance to antiandrogen therapy. Nat Med. 2004; 10: 33-9. https://doi.org/10.1038/nm972.

21. Robinson D, Van Allen EM, Wu YM, Schultz N, Lonigro RJ, Mosquera JM, Montgomery B, Taplin ME, Pritchard CC, Attard G, Beltran H, Abida W, Bradley RK, et al. Integrative Clinical Genomics of Advanced Prostate Cancer. Cell. 2015; 162:454. https://doi.org/10.1016/j. cell.2015.06.053.

22. Yanagita M, Redig AJ, Paweletz CP, Dahlberg SE, O'Connell A, Feeney N, Taibi M, Boucher D, Oxnard GR, Johnson BE, Costa DB, Jackman DM, Janne PA. A Prospective Evaluation of Circulating Tumor Cells and Cell-Free DNA in EGFR-Mutant Non-Small Cell Lung Cancer Patients Treated with Erlotinib on a Phase II Trial. Clin Cancer Res. 2016; 22: 6010-20. https://doi. org/10.1158/1078-0432.CCR-16-0909.

23. Dagogo-Jack I, Brannon AR, Ferris LA, Campbell CD, Lin JJ, Schultz KR, Ackil J, Stevens S, Dardaei L, Yoda S, Hubbeling H, Digumarthy SR, Riester M, et al. Tracking the Evolution of Resistance to ALK Tyrosine Kinase Inhibitors through Longitudinal Analysis of Circulating Tumor DNA. JCO Precis Oncol. 2018; 2018:1-14. https:// doi.org/10.1200/PO.17.00160.

24. Conteduca V, Wetterskog D, Sharabiani MT, Grande E, Fernandez-Perez MP, Jayaram A, Salvi S, Castellano D, Romanel A, Lolli C, Casadio V, Gurioli G, Amadori $\mathrm{D}$, et al, and PREMIERE Collaborators, and Spanish Oncology Genitourinary Group. Androgen receptor gene status in plasma DNA associates with worse outcome on enzalutamide or abiraterone for castration-resistant prostate cancer: a multi-institution correlative biomarker study. Ann 
Oncol. 2017; 28:1508-16. https://doi.org/10.1093/annonc/ $\operatorname{mdx} 155$.

25. Tuxen IV, Jonson L, Santoni-Rugiu E, Hasselby JP, Nielsen FC, Lassen U. Personalized oncology: genomic screening in phase 1. APMIS. 2014; 122: 723-33. https:// doi.org/10.1111/apm.12293.

26. Cancer Genome Atlas Network. Comprehensive molecular portraits of human breast tumours. Nature. 2012; 490:6170. https://doi.org/10.1038/nature11412

27. Pereira B, Chin SF, Rueda OM, Vollan HK, Provenzano E, Bardwell HA, Pugh M, Jones L, Russell R, Sammut SJ, Tsui DW, Liu B, Dawson SJ, et al. Erratum: the somatic mutation profiles of 2,433 breast cancers refine their genomic and transcriptomic landscapes. Nat Commun. 2016; 7:11908. https://doi.org/10.1038/ncomms11908.

28. Østrup O, Ahlborn LB, Lassen U, Mau-Sørensen M, Nielsen FC. Detection of copy number alterations in cellfree tumor DNA from plasma. BBA Clin. 2017; 7:120-26. https://doi.org/10.1016/j.bbacli.2017.03.006.

29. Sabari JK, Offin M, Stephens D, Ni A, Lee A, Pavlakis N, Clarke S, Diakos CI, Datta S, Tandon N, Martinez A, Myers ML, Makhnin A, et al. A Prospective Study of Circulating Tumor DNA to Guide Matched Targeted Therapy in Lung Cancers. J Natl Cancer Inst. 2018. [Epub ahead of print].

30. Ahlborn LB, Tuxen IV, Mouliere F, Kinalis S, Schmidt AY, Rohrberg KS, Santoni-Rugiu E, Nielsen FC, Lassen U, Yde CW, Oestrup O, Mau-Sorensen M. Circulating tumor DNA as a marker of treatment response in BRAF V600E mutated non-melanoma solid tumors. Oncotarget. 2018; 9:32570 79. https://doi.org/10.18632/oncotarget.25948.

31. Dawson SJ, Tsui DW, Murtaza M, Biggs H, Rueda OM, Chin SF, Dunning MJ, Gale D, Forshew T, MahlerAraujo B, Rajan S, Humphray S, Becq J, et al. Analysis of circulating tumor DNA to monitor metastatic breast cancer. N Engl J Med. 2013; 368: 1199-209. https://doi. org/10.1056/NEJMoa1213261.

32. Eisenhauer EA, Therasse P, Bogaerts J, Schwartz LH, Sargent D, Ford R, Dancey J, Arbuck S, Gwyther S, Mooney M, Rubinstein L, Shankar L, Dodd L, et al. New response evaluation criteria in solid tumours: revised RECIST guideline (version 1.1). Eur J Cancer. 2009; 45: 228-47. https://doi.org/10.1016/j.ejca.2008.10.026.

33. Diehl F, Schmidt K, Choti MA, Romans K, Goodman S, Li M, Thornton K, Agrawal N, Sokoll L, Szabo SA, Kinzler
KW, Vogelstein B, Diaz LA Jr. Circulating mutant DNA to assess tumor dynamics. Nat Med. 2008; 14: 985-90. https:// doi.org/10.1038/nm.1789.

34. Diehl F, Li M, Dressman D, He Y, Shen D, Szabo S, Diaz LA Jr, Goodman SN, David KA, Juhl H, Kinzler KW, Vogelstein B. Detection and quantification of mutations in the plasma of patients with colorectal tumors. Proc Natl Acad Sci U S A. 2005; 102: 16368-73. https://doi. org/10.1073/pnas.0507904102.

35. Mouliere F, Chandrananda D, Piskorz AM, Moore EK, Morris J, Ahlborn LB, Mair R, Goranova T, Marass F, Heider K, Wan JCM, Supernat A, Hudecova I, et al. Enhanced detection of circulating tumor DNA by fragment size analysis. Sci Transl Med. 2018; 10(466).

36. Butler TM, Johnson-Camacho K, Peto M, Wang NJ, Macey TA, Korkola JE, Koppie TM, Corless CL, Gray JW, Spellman PT. Exome Sequencing of Cell-Free DNA from Metastatic Cancer Patients Identifies Clinically Actionable Mutations Distinct from Primary Disease. PLoS One. 2015; 10: e0136407. https://doi.org/10.1371/journal. pone. 0136407 .

37. Murtaza M, Dawson SJ, Tsui DW, Gale D, Forshew T, Piskorz AM, Parkinson C, Chin SF, Kingsbury Z, Wong AS, Marass F, Humphray S, Hadfield J, et al. Non-invasive analysis of acquired resistance to cancer therapy by sequencing of plasma DNA. Nature. 2013; 497: 108-12. https://doi.org/10.1038/nature12065.

38. Ahlborn LB, Madsen M, Jonson L, Nielsen FC, Lassen U, Yde CW, Mau-Sorensen M. Concordance of Mutation Detection in Circulating Tumo r DNA in Early Clinical Trials Using Different Blood Collection Protocols. Clin Lab. 2017; 63:1755-1759.

39. Wellcome Trust Sanger Institute GRLCCosmic. http:// cancer.sanger.ac.uk/cosmic.

40. Alamut version 2.2 (Interactive Biosoftware, Rouen, France). http://www.interactive-biosoftware.com

41. Li MM, Datto M, Duncavage EJ, Kulkarni S, Lindeman NI, Roy S, Tsimberidou AM, Vnencak-Jones CL, Wolff DJ, Younes A, Nikiforova MN. Standards and Guidelines for the Interpretation and Reporting of Sequence Variants in Cancer: A Joint Consensus Recommendation of the Association for Molecular Pathology, American Society of Clinical Oncology, and College of American Pathologists. J Mol Diagn. 2017; 19: 4-23. http://cancer.sanger.ac.uk/ cosmic. 